
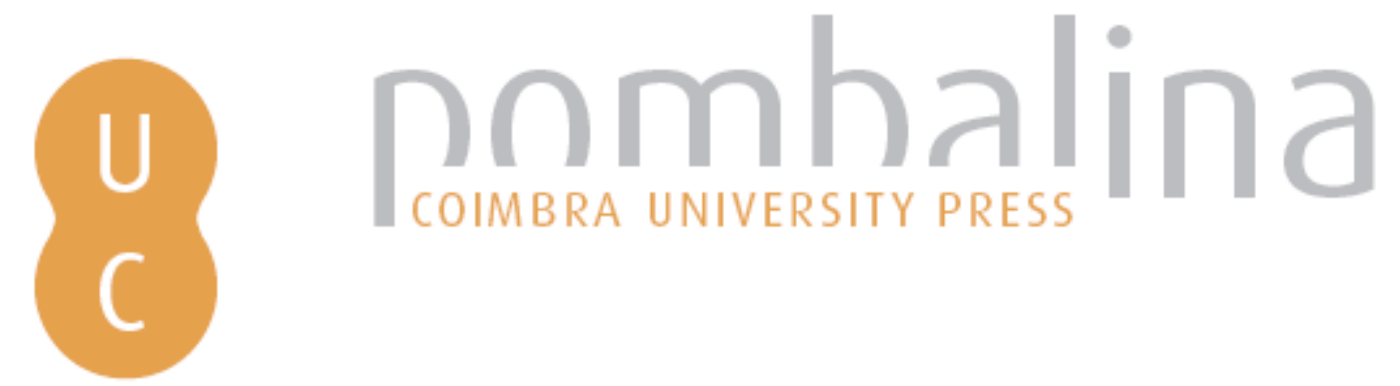

\title{
A utilização de simulações computacionais como ferramenta de apoio à prospecção geofísica com técnicas sísmicas
}

\author{
Autor(es): $\quad$ Godinho, Luís \\ Publicado por: Imprensa da Universidade de Coimbra; Laboratório de Radioactividade \\ URL \\ persistente: URI:http://hdl.handle.net/10316.2/36300 \\ DOI: $\quad$ DOI:http://dx.doi.org/10.14195/978-989-26-1009-2_13 \\ Accessed : $\quad$ 26-Apr-2023 13:52:20
}

A navegação consulta e descarregamento dos títulos inseridos nas Bibliotecas Digitais UC Digitalis, UC Pombalina e UC Impactum, pressupõem a aceitação plena e sem reservas dos Termos e Condições de Uso destas Bibliotecas Digitais, disponíveis em https://digitalis.uc.pt/pt-pt/termos.

Conforme exposto nos referidos Termos e Condições de Uso, o descarregamento de títulos de acesso restrito requer uma licença válida de autorização devendo o utilizador aceder ao(s) documento(s) a partir de um endereço de IP da instituição detentora da supramencionada licença.

Ao utilizador é apenas permitido o descarregamento para uso pessoal, pelo que o emprego do(s) título(s) descarregado(s) para outro fim, designadamente comercial, carece de autorização do respetivo autor ou editor da obra.

Na medida em que todas as obras da UC Digitalis se encontram protegidas pelo Código do Direito de Autor e Direitos Conexos e demais legislação aplicável, toda a cópia, parcial ou total, deste documento, nos casos em que é legalmente admitida, deverá conter ou fazer-se acompanhar por este aviso.

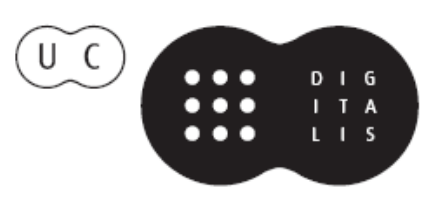




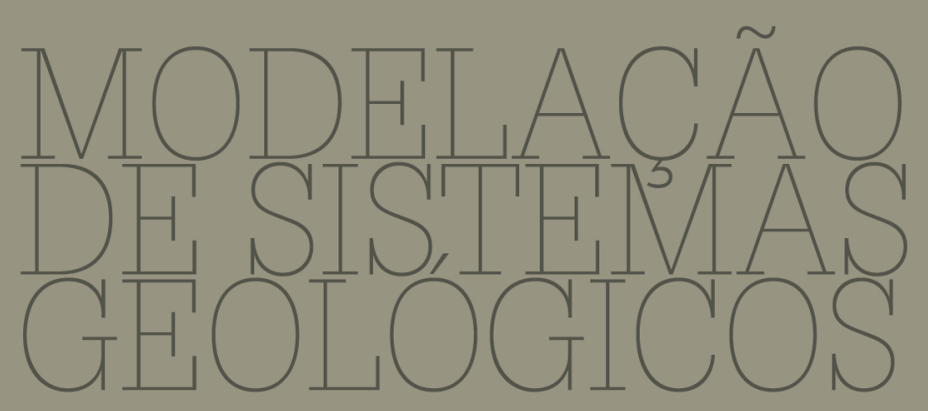

Homenagem ao Professor Doutor Manuel Maria Godinho

L.J.P.F. Neves, A.J.S.C. Pereira,

C.S.R. Gomes, L.C.G. Pereira,

A.O. TAVARES

IMPRENSA DA UNIVERSIDADE DE COIMBRA

CoImBra University Press 


\title{
MODELAÇÃO \\ DE SISTEMAS \\ GEOLÓGICOS
}

Homenagem ao Professor Manuel Maria Godinho

\section{A utilização de simulações computacionais como ferramenta de apoio à prospecção geofísica com técnicas sísmicas}

\author{
Luís Godinho ${ }^{1}$ \\ 1Departamento de Eng. Civil, Universidade de Coimbra, PORTUGAL, Igodinho@dec.uc.pt
}

Palavras-chave: Prospecção geofísica, Modelação; Sísmica; Métodos sem-malha

\section{Resumo}

Diferentes técnicas têm sido utilizadas com sucesso na prospecção geofísica de recursos naturais. Entre estas, as técnicas sísmicas têm-se revelado de grande utilidade no apoio à prospecção petrolífera, revelando-se eficazes na identificação de estruturas geológicas enterradas, essencial na previsão da localização de potenciais reservatórios petrolíferos em profundidade. Estas técnicas baseiam-se, fundamentalmente, na interpretação de sismogramas obtidos in-situ, registados num conjunto de geofones (ou hidrofones, no caso da prospecção em ambientes marítimos) devido à actuação de uma fonte sísmica. No entanto, embora a interpretação mais básica destes sismogramas forneça já informação importante sobre as estruturas existentes no local, para tirar o máximo proveito das informações recolhidas é essencial a utilização de técnicas de simulação computacional que permitam a validação e a correcção destas interpretações. Assim, o papel dos métodos numéricos aplicados à simulação da propagação de ondas acústicas e sísmicas em estruturas geológicas complexas revela-se fundamental, podendo estes constituir uma ferramenta de optimização da exploração geofísica. No presente trabalho pretende-se ilustrar a aplicação de técnicas numéricas de simulação, nomeadamente baseadas em Métodos sem Malha, no apoio à exploração sísmica de terrenos e na geração de imagens das estruturas existentes no sub-solo. Será apresentada uma breve formulação matemática das técnicas tratadas, seguindo-se um exemplo de aplicação, versando sobretudo o problema directo (simulação da propagação), mas ilustrando também os resultados que se podem obter recorrendo a técnicas de inversão.

Key-words: Meshless methods; Geophysical exploration; Complex media

\begin{abstract}
Different techniques have been used with different degrees of success in geophysical prospection of natural resources. Among those techniques, seismic methods have proved to be useful in oil prospection, allowing the accurate identification of buried structures which is essential in this task. Those methods are based, mainly, on the interpretation of seismogram acquires near the surface, at a set of geophones (or hydrophones in oceanic environments) generated by a seismic source. Although the basic interpretation of those
\end{abstract}


seismograms can, itself, provide valuable information about the existing buried structures, more sophisticated methods can greatly help to extract the maximum information from those data sets. Among those methods, numerical simulation plays a determinant role. Thus, the application of numerical techniques to simulate acoustic and elastic wave propagation in complex structures is a topic of interest for seismic exploration, which became over the last years crucial in practical applications. In this work, the application of numerical methods to simulate the propagation of acoustic waves in realistic geological scenarios is illustrated. For that purpose, a meshless formulation is presented and applied to simulate the propagation of waves in a realistic, complex model, designated as Marmousi model. The presented example aims at illustrating the application of the technique to solve the direct problem, although a preliminary result concerning the inverse (imaging) problem is also shown.

\section{Introdução}

As técnicas de prospecção geofísica são, hoje em dia, uma ferramenta essencial na identificação da estrutura geológica de terrenos, permitindo a obtenção de informação relevante sobre a variação das suas características em profundidade. Nas últimas décadas, estas técnicas têm vindo a ser progressivamente desenvolvidas e aperfeiçoadas, de forma a permitir a obtenção de informação cada vez mais detalhada sobre estas estruturas.

É comum admitir-se que estas técnicas se dividem em dois grandes grupos, inserindo-se num deles as que fazem uso dos campos gerados pela própria Terra (sejam eles eléctricos, electromagnéticos ou gravíticos) e os que requerem a introdução de energia nos terrenos recorrendo a uma ou mais fontes artificiais. Do ponto de vista prático, no primeiro grupo de técnicas avalia-se a variação dos referidos campos naturais, correlacionando-a com a presença de eventuais depósitos de recursos com potencial interesse económico; a sua principal vantagem é a de permitirem uma avaliação das características geológicas dos terrenos a maiores profundidades. No caso das técnicas que recorrem à introdução de energia com recurso a uma fonte externa, estas fontes são utilizadas para gerar localmente um campo eléctrico ou electromagnético cuja interacção com o terreno permita inferir a localização de eventuais estruturas enterradas. A grande vantagem deste tipo de técnicas consiste na maior resolução e detalhe que permitem obter, fruto de uma maior concentração de energia na zona a analisar. Neste grupo inserem-se ainda aquelas que são, talvez, as técnicas mais utilizadas no âmbito da prospecção de recursos naturais, baseadas na avaliação da propagação de ondas sísmicas e acústicas. Neste tipo de técnicas, as velocidades de propagação e os trajectos percorridos 
pelos diferentes tipos de ondas são analisadas e mapeadas em profundidade, permitindo identificar a presença e localização de estruturas reflectoras existentes em profundidade.

Diversos trabalhos de referência têm analisado a aplicabilidade, o rigor e as potencialidades de cada uma das técnicas, como os trabalhos de Keary et al. (2002) ou de Gadallah et al. (2008).

O objectivo último das técnicas de prospecção geofísica é, tal como já foi referido, a obtenção de imagens que permitam perceber, de forma concisa, a real configuração de maciços subterrâneos. Para esse efeito, a interpretação dos sismogramas obtidos pela aplicação de técnicas sísmicas torna-se essencial, em particular a correcta interpretação da posição dos impulsos reflectidos em interfaces subterrâneas registados em geofones ou hidrofones colocados à superfície. Um dos processos mais usados no apoio a esta interpretação é o da migração de dados sísmicos, apoiada em técnicas numéricas mais ou menos complexas. Neste processo, o objectivo é localizar o conjunto de pontos abaixo da superfície que produzem as reflexões registadas nos receptores superficiais, procurando ainda estimar a ordem de grandeza dos coeficientes de reflexão das superfícies que lhes correspondem.

A interpretação dos dados recolhidos usando muitas das técnicas de campo usadas na prospecção geofísica beneficia largamente do apoio adicional de ferramentas numéricas de maior ou de menor complexidade. No caso das técnicas sísmicas e acústicas, é comum o recurso a modelos numéricos de propagação de ondas para permitir uma correcta interpretação dos dados de campo. Neste domínio, as ferramentas de simulação baseadas em diferenças-finitas, em elementos finitos ou em métodos sem-malha têm vindo a ser desenvolvidas por vários investigadores. Referem-se, a título de exemplo, os trabalhos de Boore (1972), Souza et al. (2004), de Hulbert et al. (1990), de Bayliss et al. (1986) ou de Komatitsch et al. (1999) no que respeita aos métodos de elementos e diferenças finitas, e as formulações sem malha, baseadas no Element Free Galerkin Method, propostas por Jia et al. (2005, 2006).

De todos os métodos numéricos referidos, os métodos sem-malha são, seguramente, os que se encontram ainda numa fase de menor maturidade, e aos quais os investigadores têm vindo progressivamente a dedicar maior atenção. Apesar de menos desenvolvidas, estas técnicas apresentam-se muito promissoras, desde logo pelo facto de permitirem um menor esforço na definição dos modelos a simular, uma veza que, em geral, não requerem informação relativa a conectividade entre nós ou elementos. No presente trabalho pretende ilustrar-se a aplicação de uma técnica sem malha à 
simulação da propagação de ondas acústicas em meios complexos, procurando evidenciar-se a sua aplicabilidade a configurações reais. Para tal, apresenta-se neste texto uma técnica numérica sem-malha baseada na utilização de funções de base radial (RBFs) para a resolução da equação de propagação de ondas acústicas no domínio espaço-tempo. Apresenta-se, em primeiro lugar, a formulação matemática da técnica proposta, seguindo-se, depois, um exemplo de aplicação no qual se ilustra a aplicabilidade desta técnica a configurações complexas.

\section{Formulação matemática}

Para formular o problema geral de propagação de ondas num meio heterogéneo, que constitui a base das técnicas sísmicas de prospecção, considere-se um domínio generico onde a velocidade de propagação, v(x), e a densidade, $\rho(\mathbf{x})$, são dependentes do ponto do domínio considerado. Nestas circunstâncias, considerando adicionalmente que apenas se propagam ondas de compressão (caso acústico), e na ausência de amortecimento, a equação escalar de propagação de ondas pode ser escrita como

$$
\rho(\mathbf{x}) \nabla\left(\frac{1}{\rho(\mathbf{x})} \nabla \mathrm{p}(\mathbf{x}, \mathrm{t})\right)=\frac{1}{\mathrm{v}(\mathbf{x})^{2}} \frac{\partial^{2}}{\partial \mathrm{t}^{2}} \mathrm{p}(\mathbf{x}, \mathrm{t})
$$

onde $\mathrm{p}(\mathbf{x}, \mathrm{t})$ representa a grandeza avaliada no ponto $\mathbf{x}$ (que no caso acústico usualmente se considera como pressão) e no instante t. Se for considerada a simplificação adicional de um meio com velocidade constant, que não conduz a alterações nos tempos de percurso das ondas, esta equação pode ser escrita da forma simplificada

$$
\nabla^{2} \mathrm{p}(\mathbf{x}, \mathrm{t})=\frac{1}{\mathrm{v}(\mathbf{x})^{2}} \frac{\partial^{2}}{\partial \mathrm{t}^{2}} \mathrm{p}(\mathbf{x}, \mathrm{t})
$$

Esta equação de propagação estabelece a interdependência entre as derivadas temporais e espaciais da grandeza $p$, e tem que ser satisfeita em todos os pontos do domínio de propagação a analisar. Nos limites do domínio de análise torna-se ainda necessário impor as condições de fronteira adequadas, que podem corresponder à imposição de um determinado valor de $\mathrm{p}\left(\mathrm{p}(\mathbf{x}, \mathrm{t})=\mathrm{p}_{\mathrm{b}}\right)$, da sua derivada espacial numa dada direcção $\left(\frac{\partial}{\partial \mathrm{F}} \mathrm{p}(\mathbf{x}, \mathrm{t})=\mathrm{f}_{\mathrm{b}}\right)$, ou de uma relação entre estas grandezas e a derivada 
temporal de p. Este ultimo caso é particularmente útil quando se pretende impor uma condição de fronteira absorvente, como a condição de EngquistMajda de primeira ordem, dada por

$$
\frac{\partial}{\partial \mathrm{t}} \mathrm{p}(\mathbf{x}, \mathrm{t})= \pm \mathrm{v}(\mathbf{x}) \frac{\partial}{\partial \mathrm{F}} \mathrm{p}(\mathbf{x}, \mathrm{t})
$$

onde $\stackrel{\Gamma}{n}$ representa a direcção normal à fronteira no ponto de interesse.

Tal como referido na introdução deste trabalho, os métodos explícitos são, em muitos casos, preferidos para a resolução da equação (1b) por permitirem, com facilidade, o tratamento de problemas de grande dimensão. $\mathrm{O}$ uso destes métodos implica que, a priori, se conheçam as quantidades $\mathrm{p}(\mathbf{x}, \mathrm{t})$ para $\mathrm{t} \leq \mathrm{t}_{\mathrm{i}}$, e que, por isso, possam ser usadas para estimar a mesma grandeza em $t=t_{i+1}$. Considerando a estratégia clássica de marcha no tempo recorrendo a difenças finitas centradas, com um incremento temporal $\Delta \mathrm{t}$, pode escrever-se

$$
\nabla^{2} \mathrm{p}\left(\mathbf{x}, \mathrm{t}_{\mathrm{i}}\right)=\frac{1}{\mathrm{v}(\mathbf{x})^{2}}\left[\frac{\mathrm{p}\left(\mathbf{x}, \mathrm{t}_{\mathrm{i}-1}\right)-2 \mathrm{p}\left(\mathbf{x}, \mathrm{t}_{\mathrm{i}}\right)+\mathrm{p}\left(\mathbf{x}, \mathrm{t}_{\mathrm{i}+1}\right)}{\Delta \mathrm{t}^{2}}\right] .
$$

Nesta equação, o operador $\nabla^{2}=\frac{\partial^{2}}{\partial x^{2}}+\frac{\partial^{2}}{\partial y^{2}}$ depende unicamente das derivadas espaciais de $p$ no instante $t_{i}$, para o qual a distribuição de $p$ é já conhecida em todo o domínio. Assim, torna-se possível aproximar este operador usando diferentes metodologias. Destas as mais usadas, e simultaneamente mais simples, correspondem a aproximações de diferenças-finitas espaciais de diferentes ordens, como as de segunda ou de quarta ordens. Considerando, por exemplo, uma aproximação espacial de segunda ordem para este operador diferencial, pode escrever-se:

$$
\nabla^{2} \mathrm{p}\left(\mathbf{x}_{\mathrm{x}, \mathrm{y}}, \mathrm{t}_{\mathrm{i}}\right)=\left[\frac{\mathrm{p}\left(\mathbf{x}_{\mathrm{x}-\Delta x, y}, \mathrm{t}_{\mathrm{i}}\right)+\mathrm{p}\left(\mathbf{x}_{\mathrm{x}, \mathrm{y}-\Delta \mathrm{y}}, \mathrm{t}_{\mathrm{i}}\right)-4 \mathrm{p}\left(\mathbf{x}_{\mathrm{x}, \mathrm{y}}, \mathrm{t}_{\mathrm{i}}\right)+\mathrm{p}\left(\mathbf{x}_{\mathrm{x}+\Delta x, y}, \mathrm{t}_{\mathrm{i}}\right)+\mathrm{p}\left(\mathbf{x}_{\mathrm{x}, \mathrm{y}+\Delta y}, \mathrm{t}_{\mathrm{i}}\right)}{\mathrm{h}^{2}}\right]
$$

Note-se, no entanto, que esta equação apenas é válida quando se conssidera que o meio de propagação é discretizado com recurso a uma grelha regular de pontos, igualmente espaçados de $\mathrm{h}$ segundo as duas direcções ortogonais, sendo a generalização para outras configurações de pontos bastante complexa do ponto de vista da implementação computacional.

Alguns autores têm proposto procedimentos alternativos para a resolução deste problema na presença de discretizações genéricas, não regulares, do domínio, recorrendo a estratégias como o método dos elementos finitos ou a métodos sem malha (p.ex. Jia et al. (2006)). 
Recentemente, Godinho et al. (2010) propuseram uma estratégia numérica baseada num método sem malha para permitir esta análise sobre distribuições genéricas, eventualmente não regulares, de pontos. Nesta técnica, os autores usam uma representação do campo de pressões $\mathrm{p}\left(\mathbf{x}, \mathrm{t}_{\mathrm{i}}\right)$, no instante $t_{i}$, baseada numa combinação linear de funções de base radial (Radial Basis Functions - RBFs), centradas em $\mathrm{N}_{\mathrm{n}}$ pontos do domínio situados apenas na vizinhança do ponto em análise, e para o qual se pretende estimar o operador diferencial. Assim, o campo de pressões pode ser escrito como

$$
\mathrm{p}\left(\mathbf{x}, \mathrm{t}_{\mathrm{i}}\right)=\sum_{\mathrm{j}=1}^{\mathrm{N}_{\mathrm{n}}} \varphi_{\mathrm{j}}\left(\mathbf{x}_{\mathrm{j}}, \mathbf{x}\right) \mathrm{A}_{\mathrm{j}}
$$

em que $\mathbf{x}_{\mathbf{j}}$ representa o ponto central da $R B F j$. Dado que a quantidade $\mathrm{p}\left(\mathbf{x}, \mathrm{t}_{\mathrm{i}}\right)$ é conhecida para todos os pontos do domínio, torna-se possível estabelecer um sistema de $\mathrm{N}_{n}$ equações a $N_{n}$ incógnitas que permite a determinação dos factores de amplitude das funções $\left(\mathrm{A}_{\mathrm{j}}\right)$.

É importante notar que a aplicação de RBFs implica a definição de um domínio de suporte, do qual depende o número de pontos $\mathrm{N}_{\mathrm{n}}$ a considerar em (5). Caso o domínio de suporte seja muito alargado, o sistema de equações associado torna-se de grande dimensão; adicionalmente, usando as funções de base radial mais comuns, como as funções MQ (multiquádricas), o sistema torna-se totalmente preenchido, e o método exige recursos computacionais elevados, tornando-se quase impossível a sua utilização. No entanto, tendo em consideração que o objectivo da utilização desta estratégia é a estimativa do operador $\nabla^{2}=\frac{\partial^{2}}{\partial x^{2}}+\frac{\partial^{2}}{\partial y^{2}}$ (em pontos internos), ou de derivadas de primeira ordem da pressão (nos pontos da fronteira), uma aproximação local pode tornar-se adequada e proporcionar uma correcta reprodução do campo de pressões em torno de cada ponto de análise. Assim, se considerarmos um domínio de suporte mais limitado, em redor do ponto $\mathrm{k}$, incluindo apenas um número reduzido de pontos vizinhos, $\mathrm{N}_{\mathrm{n}}$ torna-se pequeno e o sistema passa a ser bastante compacto, ainda que totalmente preenchido. Apesar de compacto, este subdomínio localizado em torno do ponto de análise pode apresentar uma qualquer forma, não sendo requisito do método que os pontos que o integram se situem sobre uma grelha ortogonal. No entanto, em muitas aplicações práticas, pode ser conveniente 
a utilização desse tipo de configurações; apresentam-se, por isso, na Figura 1 exemplos dos pontos de interpolação que se obtêm nesse caso, considerando que o subdomínio apresenta uma forma circular com raios $\mathrm{h}$, $2 \mathrm{~h}$ ou $3 \mathrm{~h}$, em que h representa o espaçamento entre pontos em qualquer das direcções ortogonais.

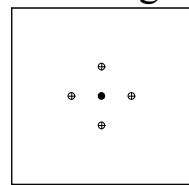

a)

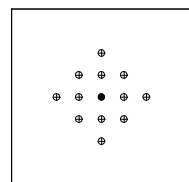

b)

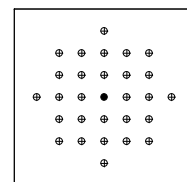

c)

Figura 1. Subdomínio local de interpolação em torno do ponto de análise quando se usam: a) 5 pontos (raio de h); b) 13 pontos (raio de 2 h); c) 29 pontos (raio de 3 h).

Dos diferentes tipos de RBFs que podem ser usados, as do tipo MQ são, provavelmente, as mais utilizadas, assumindo a forma

$$
\varphi\left(\mathbf{x}_{\mathrm{i}}, \mathbf{x}_{\mathrm{j}}\right)=\sqrt{\mathrm{r}^{2}+\mathrm{c}^{2}}, \operatorname{com} \mathrm{r}=\sqrt{\left(\mathrm{x}_{\mathrm{j}}-\mathrm{x}_{\mathrm{i}}\right)^{2}+\left(\mathrm{y}_{\mathrm{j}}-\mathrm{y}_{\mathrm{i}}\right)^{2}} \text {, para } \mathbf{x}_{\mathrm{i}}, \mathbf{x}_{\mathbf{j}} \in \Omega_{\mathrm{k}},
$$

onde $\Omega_{\mathrm{k}}$ é o domínio local de análise em torno de $\mathrm{x}_{\mathrm{k}}$, e $c$ é o parâmetro livre (ou parâmetro de forma) da função MQ. Este parâmetro pode afectar grandemente o rigor da aproximação pretendida, e a sua selecção criteriosa ainda é um problema em estudo por muitos investigadores. (veja-se, por exemplo, os trabalhos de Kansa e Hon (2000), Sarra e Sturgill (2009), ou Cheng et al. (2003)). Devido à dificuldade em encontrar um valor óptimo para este parâmetro, outras funções, como as da família $\mathrm{r}^{2 n+1}$, têm também sido testadas.

Para o caso específico da propagação de ondas em sistemas acústicos, Godinho et al. (2010) propuseram uma estratégia que permite estimar um valor adequado para o parâmetro livre da função $\mathrm{MQ}$, tendo em consideração alguns pressupostos aplicáveis a esse tipo de problema. Assim, supondo que a onda que se propaga no sistema apresenta a configuração de um pulso de Ricker (dada, no domínio do tempo, pela função $\mathrm{p}(\tau)=\left(1-2 \tau^{2}\right) \mathrm{e}^{-\tau^{2}}$, com $\tau=\left(\mathrm{t}-\mathrm{t}_{\mathrm{s}}\right) / \mathrm{t}_{0}$, sendo $\mathrm{t}_{\mathrm{s}} \mathrm{o}$ tempo para o qual ocorre $\mathrm{o}$ valor de pico e $\mathrm{f}_{0}=1 / \pi \mathrm{t}_{0}$ a frequência característica do pulso), estes autores concluiram que um valor adequado para c será o que melhor permite reproduzir as segundas derivadas deste pulso quando ele é aplicado espacialmente em torno do ponto de análise. Para o caso de malhas regulares, e para a situação em que o raio que define o subdomínio é $2 \mathrm{~h}$, os autores concluiram que o parâmetro c pode ser estimado a partir da curva apresentada na Figura 2. 


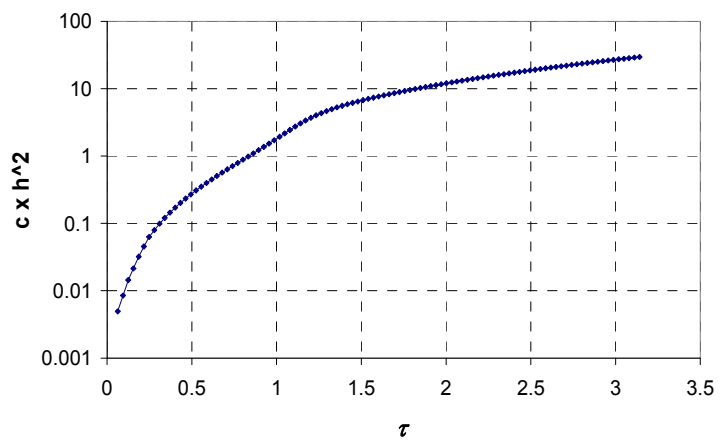

Figura 2. Curva que traduz o valor de c para malhas regulares com espaçamento $\mathrm{h}$, sendo $\tau=f_{0} h / v_{0}$.

\section{Aplicação a um sistema geológico complexo - o modelo de Marmousi}

Para ilustrar as potencialidades dos modelos matemáticos referidos na secção anterior, procedeu-se à sua aplicação para a simulação da propagação de ondas geradas por uma fonte de pressão na presença de um sistema geológico complexo. Para este efeito, considere-se a configuração tratada em Versteeg (1994), designada usualmente por modelo de Marmousi. Tratase de um modelo que representa um ambiente marinho e que reproduz uma zona específica da Bacia de Cuanza, em Angola.

Do ponto de vista geológico, a formação desta estrutura pode separarse em duas fases distintas. Numa primeira fase ocorreram fenómenos de sedimentação predominantemente de margas e carbonatos. Seguiu-se uma fase em que estes depósitos foram ligeiramente dobrados e erodidos, gerando uma superfície final aproximadamente plana. Numa segunda fase ocorreu um conjunto de fenómenos de evaporação, dando origem a um depósito de sal sobre a superfície plana referida. Nesta sequência, depositaram-se, também, argilas margosas ricas em matérias orgânicas. Seguiu-se então a deposição de sedimentos argilo-arenosos, em camadas espessas, cuja pressão originou o deslizamento lateral do depósito de sal subjacente. Neste processo de deslizamento do sal originaram-se falhas geológicas que poderão ter estado activas durante um período prolongado, gerando uma descontinuidade entre os estratos das camadas superiores. A Figura 3 apresenta uma representação esquemática deste modelo, ilustrando as velocidades de propagação das ondas de compressão associadas a cada uma das camadas. Assim, no modelo de Marmousi, a camada superior corresponde à água do mar, com uma velocidade de propagação de cerca de $1500 \mathrm{~m} / \mathrm{s}$. Abaixo da água, sucedem-se diferentes camadas com 
características distintas, representando estratos mais ou menos rígidos. A uma profundidade entre $2 \mathrm{~km}$ e $3 \mathrm{~km}$ surge a camada de sal, material muito rígido e onde se atingem velocidades de propagação das ondas de compressão de cerca de $5500 \mathrm{~m} / \mathrm{s}$.

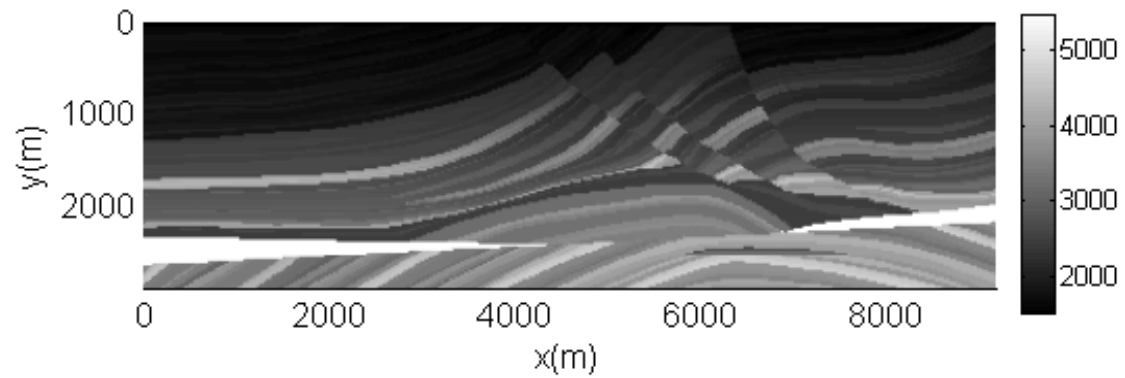

Figura 3. Distribuição de velocidades de propagação das ondas de compressão no modelo de Marmousi.

Do ponto de vista geofísico, esta estrutura geológica apresenta uma enorme complexidade, sendo expectáveis padrões de propagação de ondas de difícil interpretação. No caso de se considerarem modelos de simulação de ondas elásticas, essa complexidade é ainda maior. Por esse motivo, grande parte das análises encontradas na literatura fazem uso de modelos de propagação de ondas acústicas, por serem de mais simples interpretação, mantendo, ainda assim, muitas das características fundamentais dos fenómenos físicos registados in-situ. No presente trabalho aplica-se o método sem-malha descrito na secção anterior para simular a propagação de ondas acústicas no referido modelo. Para esse efeito, o modelo original foi reamostrado numa grelha de pontos distribuídos de forma regular no espaço, com espaçamento de $12 \mathrm{~m}$ nas duas direcções ortogonais, totalizando 221540 pontos. Todos os resultados aqui apresentados foram obtidos recorrendo a marcha no domínio do tempo com intervalos de $0.5 \mathrm{~ms}$.

Na Figura 4 apresentam-se três "snapshots" que representam a pressão acústica calculada pelo modelo proposto em três momentos distintos, após disparo de uma fonte localizada em $\mathrm{x}=2928 \mathrm{~m}$ e $\mathrm{y}=72 \mathrm{~m}$.

No instante inicial, $\mathrm{t}=0.5 \mathrm{~s}$, o padrão de propagação apresenta ainda um conjunto reduzido de frentes de onda, uma vez que, até este instante, a propagação se deu essencialmente na água e nas camadas sedimentares mais superficiais. Assim, torna-se claramente identificável a onda incidente, seguida de uma frente de onda com a fase invertida, correspondente a uma primeira reflexão ocorrida na superfície livre. À medida que o tempo 
avança, a energia propaga-se afastando-se do ponto fonte. Nesse processo, atinge as restantes camadas do modelo, onde se geram novas reflexões. $\mathrm{O}$ padrão torna-se, por isso, mais complexo, e para $\mathrm{t}=1.0 \mathrm{~s}$ registam-se

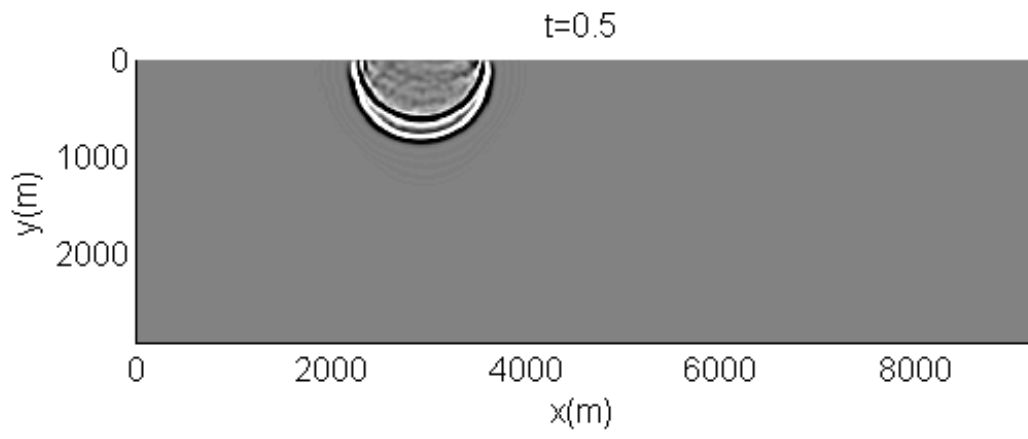

a)

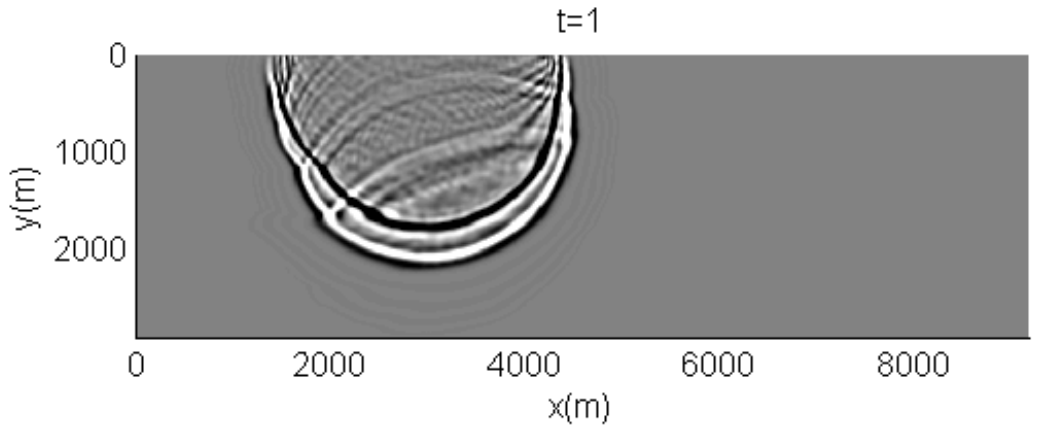

b)

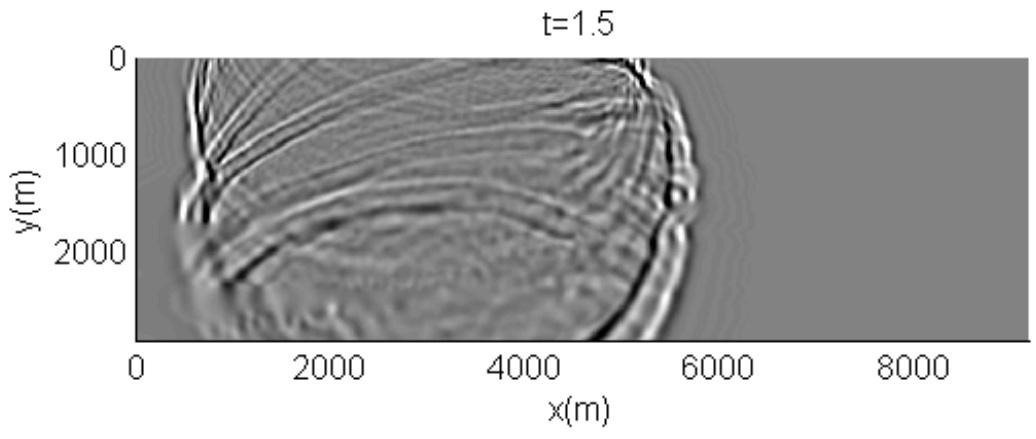

c)

Figura 4. "Snapshots" da propagação de ondas no modelo acústico.

claramente frentes de onda adicionais originadas em reflexões da energia incidente em interfaces de maior contraste. Note-se, na mesma imagem, que 
a forma inicialmente circular da frente de onda incidente vai progressivamente dando lugar a uma geometria mais complexa, situação que é gerada pela existência de velocidades de propagação distintas nas diferentes camadas do modelo. Para $t=1.5 \mathrm{~s}$ a energia atingiu já a profundidade máxima do modelo, e o padrão de propagação integra agora um elevado número de frentes de onda. O efeito da presença de uma camada rígida (sal) torna-se agora evidente, levando a que ocorra uma grande atenuação da energia que se propaga a camadas situadas a maiores profundidades. É, no entanto, interessante verificar que, na zona central do modelo, a descontinuidade das camadas de sal permite que a onda incidente atinja as camadas inferiores (sob o sal) com maior amplitude.

A análise até aqui apresentada pretendeu, apenas, ilustrar a aplicabilidade do método numérico proposto ao estudo da propagação de ondas em meios de grande complexidade. Do ponto de vista da prospecção geofísica, o maior interesse está, no entanto, na obtenção de imagens em profundidade que permitam, a partir de uma estimativa inicial, obter informações relativas à possível localização e dimensão de reservatórios de recursos fósseis (nomeadamente petróleo). Para o efeito, é comum juntar aos modelos de propagação, como o que aqui se apresentou, métodos de migração sísmica, que permitam a obtenção de imagens fidedignas das estruturas enterradas. Na Figura 6 ilustra-se o resultado da aplicação de uma técnica de migração de dados sísmicos, a "RTM - reverse time migration" (migração reversa no tempo), em conjunto com o modelo de propagação proposto ao modelo geológico de Marmousi. Para a aplicação desta metodologia, consideraram-se várias posições de fonte distintas, espaçadas entre si de $100 \mathrm{~m}$, todas elas a $72 \mathrm{~m}$ de profundidade, e realizou-se o cálculo dos sismogramas sintéticos em receptores localizados junto da superfície. $\mathrm{O}$ processo de migração foi efectuado através do empilhamento das imagens obtidas após migração ("empilhamento pós-migração"), não tendo, no entanto, sido aplicados nenhuns mecanismos de aperfeiçoamento da imagem ou de processamento adicional. A imagem assim obtida (Figura 5) revela, ainda assim, o aspecto geral do modelo de Marmousi, sendo claramente identificáveis as várias camadas que o compõem. É interessante notar que, na zona central, entre as duas estruturas de sal, se observa a presença de uma pequena zona mais escura, que corresponde a um reservatório enterrado (que pode também ser claramente identificado no modelo inicial da Figura $3)$. 


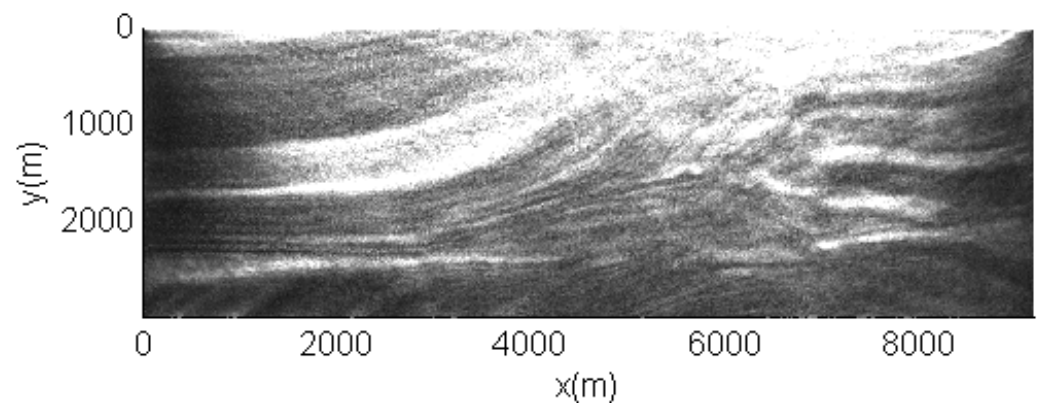

Figura 5. Imagem em profundidade obtida por migração (RTM) de sismogramas sintéticos.

\section{Conclusões}

No presente trabalho apresentou-se a formulação matemática de um método sem malha que permite a simulação da propagação de ondas acústicas em sistemas geológicos complexos. Ilustrou-se, adicionalmente, a sua aplicação a um dos casos de teste mais divulgados na área da exploração petrolífera, o modelo de Marmousi, mostrando que o método proposto é facilmente aplicável a problemas de elevada complexidade. Para além dos casos apresentados neste trabalho, torna-se importante referir que, por se tratar de uma técnica sem-malha, a formulação proposta pode apresentar vantagens significativas em relação a outras técnicas, nomeadamente por permitir a adopção de distribuições não regulares de pontos (ao contrário das técnicas clássicas de diferenças-finitas), e por não necessitar de informação sobre conectividades entre elementos (ao contrário do método dos elementosfinitos), vantagens que podem facilitar a criação de modelos complexos incluindo, por exemplo, fronteiras irregulares.

\section{Bibliografia}

Bayliss, A., Jordan, K.E., Lemesurier, B.J., Turkel, E. (1986) - A fourth-order accurate finitedifference scheme for the computation of elastic waves. Bulletin of the Seismological Society of America; 76(4), 1115-1132.

Boore, D. (1972) - Finite-difference methods for seismic waves. Methods in computational physics (Ed. B. Bolt); 11, 1-37, New York.

Cheng, A. H.-D., Golberg, M.A., Kansa, E.J., Zammito, G. (2003) - Exponential convergence and hc Multiquadric collocation method for partial differential equations. Numer. Meth. Partial Diff. Eq.; 19, 571-594.

Gadallah, M. R., Fisher, R. (2009) - Exploration Geophysics. Springer-Verlag.

Godinho, L., Dors, C., Soares Jr., D., Amado-Mendes, P. (2010) -A Local Radial Basis Function Interpolation Model to Simulate Time-Domain Acoustic Wave Propagation". The Tenth 
International Conference on Computational Structures Technology, Valencia, Espanha, Setembro de 2010.

Hulbert, G., Hughes, T. (1990) - Space-Time finite Element Methods for Second-order Hyperbolic Equations. Computer Methods in Applied Mechanics and Engineering; 84, 327-348.

Jia, X. Hu, T. (2006) - Element-free precise integration method and its applications in seismic modelling and imaging, Geophys. J. Int.; 166, 349-372.

Jia, X. Hu, T., Wang, R. (2005) - A Meshless Method for Acoustic and Elastic Modeling, Appl. Geophys.; 2, 1-6.

Kansa, E., Hon, Y.C. (2000) - Circumventing the ill-conditioning problem with multiquadric radial basis functions: Applications to elliptic partial differential equations. Comput. Math. Appl.; 39, 123-137.

Kearey, P., Brooks, M., Hill, I. (2002) - An Introduction to Geophysical Exploration. Blackwell Science.

Komatitsch, D., Tromp, J. (1999) - Introduction to the spectral element method for three-dimensional seismic wave propagation. Geophysical Journal International; 139 (3), 806-822.

Sarra, S.A., Sturgill, D. (2009) - A random variable shape parameter strategy for radial basis function approximation methods. Eng. Anal. Boundary Elem.; 33, 1239-1245.

Souza, L. A., Carrer, J., Martins, C. (2004) - A Fourth Order Finite Difference Method Applied to Elastodynamics: Finite Element and Boundary Element Formulations. Structural Engineering and Mechanics; 17, 735-749.

Versteeg, R. (1994) - The Marmousi experience: Velocity model determination on a synthetic complex data set. The Leading Edge; 927-936. 